\title{
SCORE Imaging: Specimen in a Corrected Optical Rotational Enclosure
}

\author{
Andrew M. Petzold,,2 Victoria M. Bedell, ${ }^{1}$ Nicole J. Boczek, ${ }^{2,3}$ Jeffrey J. Essner, ${ }^{4}$ \\ Darius Balciunas, ${ }^{1,5}$ Karl J. Clark, ${ }^{2}$ and Stephen C. Ekker ${ }^{1,2}$
}

\begin{abstract}
Visual data collection is paramount for the majority of scientific research. The added transparency of the zebrafish (Danio rerio) allows for a greater detail of complex biological research that accompanies seemingly simple observational tools. We developed a visual data analysis and collection approach that takes advantage of the cylindrical nature of the zebrafish allowing for an efficient and effective method for image capture that we call Specimen in a Corrected Optical Rotational Enclosure imaging. To achieve a nondistorted image, zebrafish were placed in a fluorinated ethylene propylene tube with a surrounding optically corrected imaging solution (water). By similarly matching the refractive index of the housing (fluorinated ethylene propylene tubing) to that of the inner liquid and outer liquid (water), distortion was markedly reduced, producing a crisp imagable specimen that is able to be fully rotated $360^{\circ}$. A similar procedure was established for fixed zebrafish embryos using convenient, readily available borosilicate capillaries surrounded by $75 \%$ glycerol. The method described here could be applied to chemical genetic screening and other related high-throughput methods within the fish community and among other scientific fields.
\end{abstract}

\section{Introduction}

$\mathbf{V}$ ISUAL DATA CAPTURE is a major part of scientific documentation. The transparency of the zebrafish (Danio rerio) has been an invaluable backdrop to the use of this system for complex biological research based on relatively simple and classically optical observation. One of the major hurdles of the zebrafish researcher has been the capture and meaningful representation of this visual data. Many aspects of zebrafish biology are better expressed in images and other digital media rather than in written descriptions. Examples include assessment of morphological mutant phenotypes, protein or nucleic acid expression patterns, and developmental stages. ${ }^{1-3}$

Part of the challenge in obtaining consistent, high-quality images of the zebrafish arises from efforts in attaining static two-dimensional images of a dynamic three-dimensional sample. Additional difficulty comes from the shift of a largely spherical early embryo (0-24 hour postfertilization [hpf ]) to the cylindrical shape of the larvae and adult fish. Traditionally, this problem has been addressed by sectioning the fish either physically or optically. However, preparation of the sample is laborious and the depth of field can be lost. To further increase the photographic challenge, beginning at $72 \mathrm{hpf}$ the zebrafish larva inflates its swim bladder, ${ }^{3}$ providing buoyancy, a challenge that adds difficulty in manipulating and immobilizing specimens in a purely sagittal or coronal plane (Cartoon, Fig. 2B). The issues of working with this unique shape have inspired many methods that attempt to remedy these problems and deliver consistent, high-quality image capture. ${ }^{1-4}$ However, all of these approaches still require substantial training to achieve proficiency, are laborious, and thus are often not practical for high-throughput screening or other applications. Quality imaging can be the most critical bottleneck in using the fish for many scientific areas.

We developed a simple and efficient method for holding and photographing embryonic and larval zebrafish that we termed Specimen in a Corrected Optical Rotational Enclosure (SCORE) imaging. The SCORE method takes advantage of the cylindrical nature of an embryo and allows a specimen to be freely rotated about its longitudinal axis. This rotation, in

\footnotetext{
${ }^{1}$ Comparative and Molecular Biology, University of Minnesota, Minneapolis, Minnesota.

${ }^{2}$ Department of Biochemistry and Molecular Biology, Mayo Clinic, Rochester, Minnesota.

${ }^{3}$ Department of Biology, Saint Olaf College, Northfield, Minnesota.

${ }^{4}$ Department of Genetics, Development, and Cell Biology, Iowa State University, Ames, Iowa.

${ }^{5}$ Department of Biology, Temple University, Philadelphia, Pennsylvania.
} 

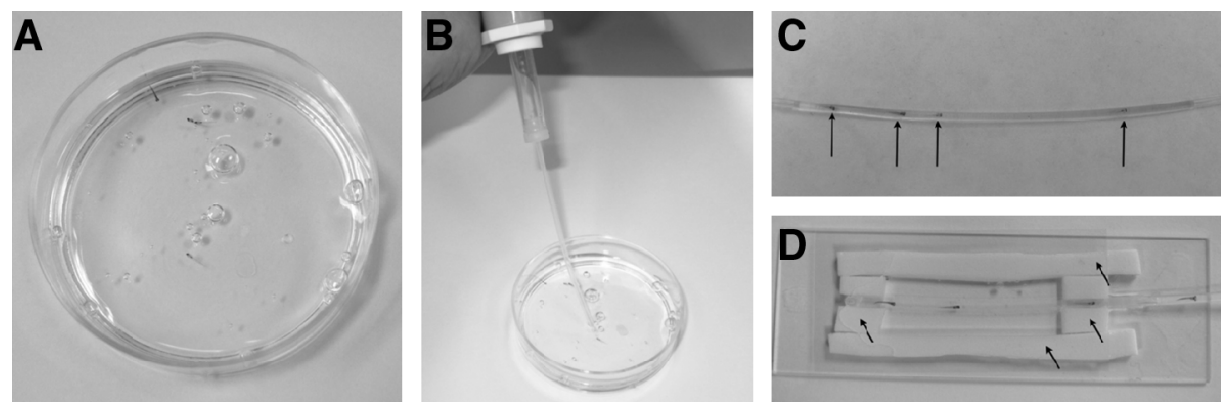

FIG. 1. SCORE tools. (A) Anesthetized larval zebrafish are placed into a methylcellulose (pictured) or low-melting-point agarose solution before being loaded into the capillary housing. (B) A pipette pump with capillary adaptor is used to facilitate loading of zebrafish into the capillary housing. (C) Multiple larvae (arrows) can be imaged in a single capillary, allowing for rapid screening of mutants. (D) A corrective solution (water pictured) is placed upon the capillary to reduce distortion. Foam barriers, marked by arrows, are placed upon the glass slide to help contain corrective solution and to provide a friction surface for ease of rotation.

turn, facilitates image capture of sagittal, coronal, or other horizontally angled planes. To accomplish the rotation, larvae are drawn into either a polymer or a borosilicate capillary housing, and are immobilized using a viscous liquid either low melting point (LMP) agarose or methylcellulose for living larvae or glycerol for fixed embryos. If the fixed embryos are placed in a permanent mounting solution, it is possible that these embryos can be stored indefinitely. The SCORE method uses a capillary tube to allow rotation about the longitudinal axis and combines it with a solution that matches the refractive index of the capillary to eliminate distortion. This process allows the user to quickly obtain publication-quality images in multiple, perfectly aligned imaging planes.

\section{Materials and Methods}

\section{Immobilization of the zebrafish embryos}

For all of our procedures, we have used the anesthetic tricaine (MS-222) at standard concentrations. ${ }^{5}$ Fish were further immobilized using either a $2 \%$ methylcellulose solution or an LMP agarose solution (at $\sim 0.5 \%$ in embryo water). If fixed larvae were imaged (such as from a whole-mount in situ hybridization [WISH] experiment), a 75\% glycerol solution was used due to its viscosity and similar refractive index to that of borosilicate glass.

\section{Preparation for SCORE imaging}

After fish were immobilized (Fig. 1A), a capillary adapter was placed in a pipette pump and used to draw an embryo into an appropriate-sized borosilicate capillary (Fig. 1B). When using LMP agarose, the capillary is then allowed to rest for $2 \mathrm{~min}$, letting the LMP agarose solidify. Multiple fish can be loaded into a single capillary, allowing for greater variety in sample choice and for data analyses (Fig. 1C). Note that the inner liquid must almost fill the entire capillary to lessen the chance of mixing with the potentially different external optical imaging solution. A paraffin wax plug can be added to the end of the capillary by way of scraping or a stick-and-twist method to block this possible contamination and to eliminate sample drift.

\section{Choice of capillary size}

Embryos begin as a bulbous shape and straighten as they age. Consequently, the size of the capillary opening depends inversely on the age of embryonic, larval, and young fish. Capillaries with a $0.60 \mathrm{~mm}$ inside diameter work well for $48+\mathrm{hpf}$ embryos (WPI \#1B120-3 or WPI \#TW100-3 for thin-walled applications). For younger embryos (0-48 hpf), an inside diameter of at least $1.12 \mathrm{~mm}$ was used (WPI \#TW150-3). When choosing the size of the polymer tubing, the smallest readily available tubing (inside diameter of $0.8 \mathrm{~mm}$; ColeParmer EW-06406-60) was used for all embryos $24 \mathrm{hpf}$ and older; however, all younger embryos needed to be dechorionated. Within the size range of the capillaries tested, the outer diameter of the capillary seems not to factor in any distortion of the embryo; however, the width of the wall of the capillary may. Thus, it is recommended that thin-walled capillaries be used when possible.

\section{Choice of plastic tubing to match refractive index of water}

We tested a variety of plastic tubing to best match the refractive index of the tubing to that of water for the greatest ease of use and image clarity. We determined that fluorinated ethylene propylene (FEP) tubing is optimal for use with water as an optical imaging solution and either LMP agarose or methylcellulose as an immobilization medium (Table 1).

\section{Matching the refractive index of borosilicate glass using optical imaging solutions}

We tested a variety of imaging solutions to match the refractive index of borosilicate glass. We determined that a $75 \%$ glycerol solution is best for use when paired with fixed embryos. Due to a fear of harm to a living embryo, a 50\% sucrose solution is recommended if it is preferred to use a borosilicate capillary over plastic tubing. A more in-depth analysis of these choices is presented in Table 2.

\section{SCORE imaging}

The capillary containing the sample is placed onto a slide with a channel with edges at least as high as the outer diameter of the capillary, allowing at least one end of the capillary to extend beyond this channel to allow rotation. This barrier can be made by affixing capillaries of a slightly larger diameter, or craft foam (Crafts Etc, $2 \mathrm{~mm}$ Funky Foam) onto a glass slide. The appropriate optical imaging solution (water, $50 \%$ sucrose, or $75 \%$ glycerol) should be applied over the 
Table 1. Refractive Indices of Polymer Tubing Used

\begin{tabular}{lcl}
\hline Solution & Refractive index & \multicolumn{1}{c}{ Comments about use } \\
\hline Water (surrounding fluid) & $1.34^{15}$ & Used to cover tubing; matched for tubing \\
FEP & $1.338^{16}$ & Recommended for live embryos \\
PFA & $1.35^{17}$ & Works well for live embryos \\
PTFE & $1.35-1.38^{16}$ & Not recommended \\
\hline
\end{tabular}

FEP, fluorinated ethylene propylene; PFA, perfluoroalkoxy; PTFE, polytetrafluoroethylene.

capillary so that the entire height of the capillary is covered (Fig. 1D). Care must be taken to ensure that no excess optical imaging solution spills onto the microscope optics. A cover slip is then placed on top of the capillary immersed in the corrective solution. Once the cover slip has been deployed, the slide can be examined under a microscope. The orientation of the embryo can be adjusted by simply rotating the capillary, the ends of which extend past the viewing area. The slides may be reused after the images have been obtained if cleaned fully. To recover the embryos from the capillary, carefully snap off the wax plugs and expel the embryos by applying pressure to the side of the capillary. All rotation of the capillary is based off of $0^{\circ}$ being a coronal image with the dorsal side upward and the lens of the specimen aligned, from this mark, sagittal is defined as a $90^{\circ}$ counterclockwise rotation and ventral is defined as a $180^{\circ}$ rotation. Any other rotational angle is approximate and is noted as such.

\section{Digital photography and editing}

Images were obtained using a Nikon D3 (Fig. 2), a Canon Powershot A640 digital camera (Fig. 3), a Zeiss ApoTome Grid Microscope (Fig. 4), or a Canon G10 (Figs. 1 and 5). Videos were captured using a Sony HDR-HC9 video camera and edited using iMovie 09 (Apple, Inc.). Bright-field images were captured using a shutter speed of 1/200 (Figs. 2 and 3). Green fluorescent photographs were captured using a shutter speed of 1/60 (Fig. 2). Red fluorescent photographs were captured using a shutter speed of 1/25 (Fig. 2). Photographs were edited with Keynote 09 for Macintosh OSX (Apple, Inc.) to allow for differing focal planes and to remove shadow caused by the capillary housing; no parts of the image of the fish were removed in this editing process.

\section{Zebrafish collection and preservation}

Embryos were collected at day zero, $\sim 1 \mathrm{hpf}$, and placed into $100 \times 15 \mathrm{~mm}$ Petri dishes with no more than 100 embryos per dish. These dishes were then stored in a $30^{\circ} \mathrm{C}$ incubator. At 3 day postfertilization, larvae were then transferred into $60 \times 15 \mathrm{~mm}$ Petri dishes with no more than 20 fish per dish for fluorescent-based genotyping according to Davidson et al. ${ }^{6}$

\section{Transgenic fish}

The doubly transgenic $\operatorname{Tg}($ fli-1:eGFP $) \mathrm{y} 1^{7} / \operatorname{Tg}($ gata-1:DsRed $)$ $\mathrm{sd} 2^{8}$ fish were as described by Pickart et al. ${ }^{9}$ Protein trap fish line $\operatorname{Tg}($ SEC0124)mn124 shown in Figure 4 was generated in a similar manner to that described previously. ${ }^{10}$ Zebrafish were injected with $25 \mathrm{ng}$ of plasmid containing the gene-break protein trap transposon GBT-R14.5. This construct is the same as GBT-R15 ${ }^{11}$ with a full-length Tol2 right inverted terminal repeat instead of the mini Tol2 end found in GBT-R15.

\section{Microscope ease of use}

To facilitate longer hours on a fluorescent microscope required for high-throughput screening without producing the eyestrain of a traditional microscope, we employed the camera port of an inverted Zeiss fluorescent microscope to its fullest potential by connecting a Sony HDR-HC9 highdefinition video camera (camera, Fig. 5A; microscope, Fig. $5 \mathrm{~B})$. The output of the high-definition video camera can be run directly into either a video screen (Fig. 5C) or a computer for video capture (Fig. 5D). Notably, we have removed the eyepieces of the microscope to ensure that no unwanted light bleaches the image when working with fluorescence (Fig. 5E) and to vastly improve access to the samples (Fig. 5F). This microscope setup also allows colleagues to easily and simultaneously observe phenotypes and patterning in the zebrafish, an environment that facilitates scientific discussion in real time. Although not necessary for the SCORE approach, this microscope setup vastly improves the accessibility to embryos in SCORE and eases high-throughput imaging.

Table 2. Refractive Indices of Borosilicate Glass Capillaries and Optical Imaging Solutions Used

\begin{tabular}{lcl}
\hline Solution & Refractive index & \multicolumn{1}{c}{ Comments about use } \\
\hline Borosilicate glass capillary & $1.47-1.49^{14}$ & Used for housing \\
Water & $1.34^{15}$ & Slight distortion, but can work if needed (not shown) \\
Vegetable oil (soybean) & $1.47^{14}$ & Not much distortion; slight GFP background \\
Mineral oil & $1.48^{14}$ & No distortion creates bubbles easily \\
$75 \%$ Glycerol & 1.46 & High viscosity; may harm living fish; best for fixed larvae \\
Light corn syrup (Brix $=80)$ & $\sim 1.5^{18}$ & High viscosity \\
$50 \%$ Sucrose & $1.464^{18}$ & Recommended optical imaging solution for live embryos \\
& & if using borosilicate glass
\end{tabular}

Brix, measurement of the fraction of sugar per hundred parts of aqueous solution as measured by mass. 
FIG. 2. Specimen in a Corrected Optical Rotational Enclosure (SCORE) imaging of living animals. Zebrafish imaging is facilitated by use of a fluoro-carbon tube for housing. (A) Zebrafish images taken in polymer tubing produce a distorted image (arrows) due to the change in refractive index between the tubing and the air. Cartoon images representing a fish immobilized using traditional techniques on a skewed plane (B), a larval fish within a polymer tube without a corrective solution producing distortion $(\mathbf{C})$, and within a corrective solution to produce a clear image (D). Fluorinated ethylene propylene (FEP) tubing produces a clear image at $50 \times($ E ) (also see Supplemental Movie S1, available online at www.liebertonline.com), $100 \times(\mathbf{F})$, and $200 \times(\mathbf{G})$ of a 5 day postfertilization (dpf) larvae. A $5 \mathrm{dpf} \mathrm{Tg}($ gata-1:DsRed)sd2 larvae in FEP tubing produces a clear background-free image at $50 \times(\mathbf{H})$ (also see Supplemental Movie S2, available online at www liebertonline.com) and $100 \times$ (I). A 5 dpf $\mathrm{Tg}($ fli:eGFP)y1 in FEP tubing produces a clear background-free image at $50 \times(\mathrm{J})$ (also see Supplemental Movie S3, available online at www.liebertonline.com) and $100 \times(\mathbf{K})$.
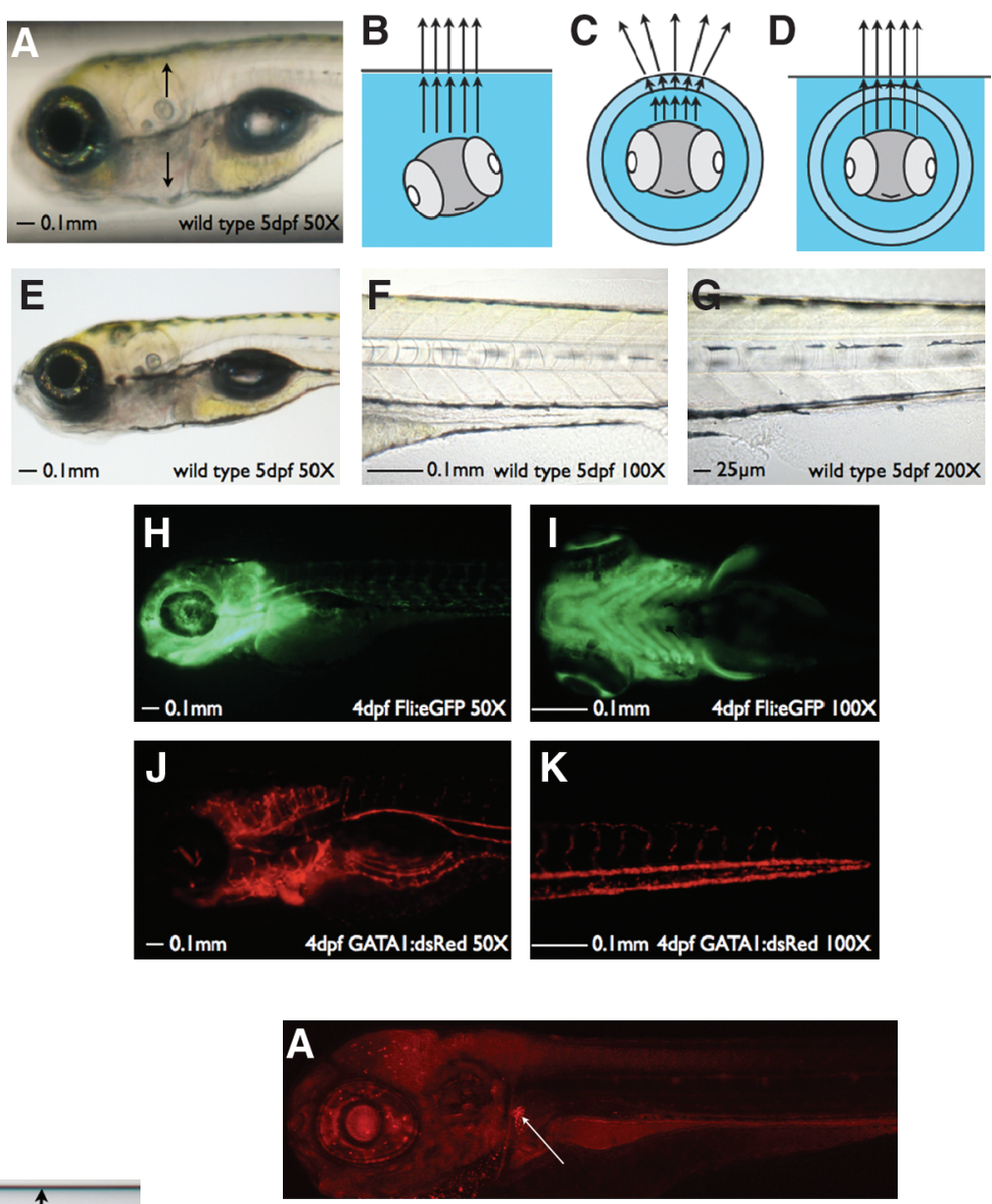

A
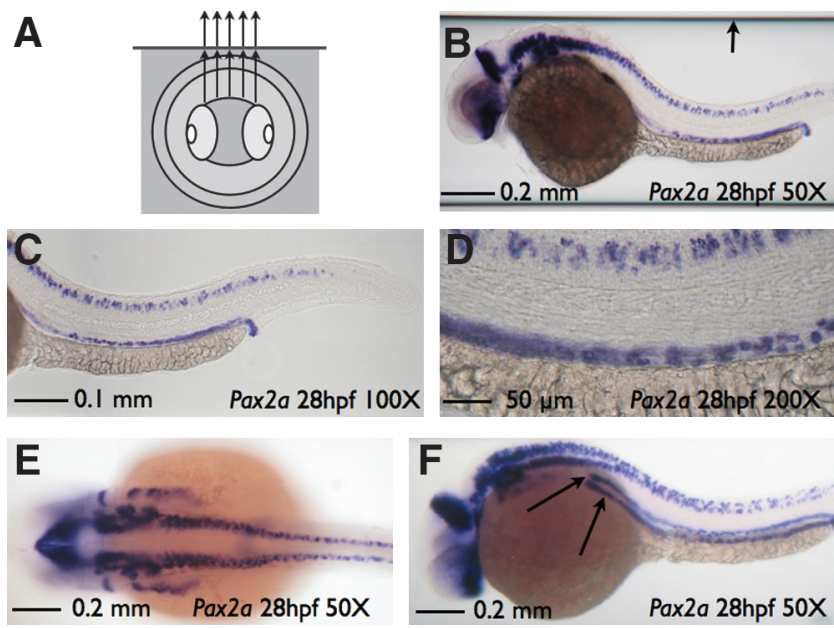

FIG. 3. SCORE imaging of a fixed specimen. Imaging of a pax $2 a$ whole-mount in situ hybridization embryo and capillary housing in $75 \%$ glycerol. (A) Cartoon depicting the use of glycerol both inside and outside of the glass tube producing an undistorted image of a fixed embryo. (B) Sagittal image of embryo with pax2 staining at $50 \times$ magnification shows no distortion (also see Supplemental Movie S4, available online at www.liebertonline.com). Note the edges of the capillary (arrow) that can be readily cropped for publication presentation. Sagittal embryo image of pax2 staining at $100 \times(\mathbf{C})$ or $200 \times($ D) magnification shows no distortion. (E) Coronal image of embryo at $50 \times$ magnification. (F) Angled image of pax 2 staining at $50 \times$ magnification. Rotation is angled slightly $\left(\sim 30^{\circ}\right)$ to show a more detailed view of kidney tubule staining (arrows).
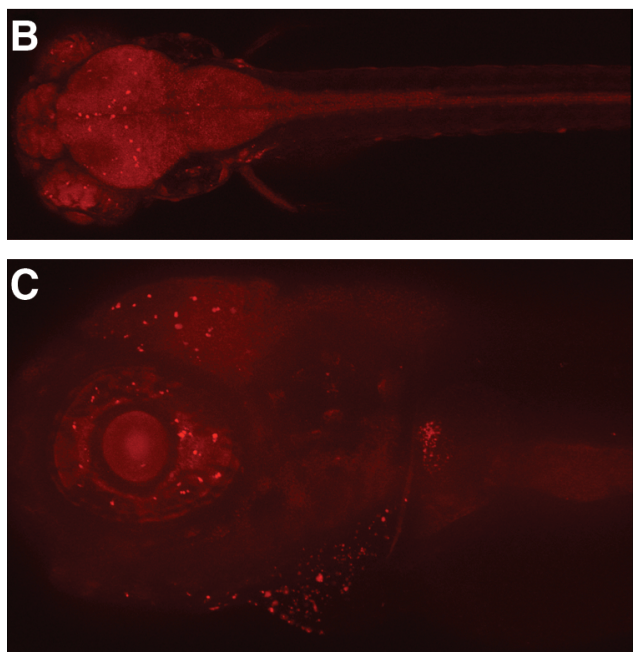

FIG. 4. SCORE imaging of fluorescent, living zebrafish. Imaging SEC0124 of a $5 \mathrm{dpf}$ mRFP-labeled protein trap zebrafish housed in FEP tubing. (A) Sagittal image of an mRFP-labeled protein trap zebrafish shows enhancement in specific locations in the neural region as well as a bright kidney (Arrow). (B) Coronal image of an mRFP-labeled protein trap zebrafish includes ubiquitous neural expression with enhancement in specific locations. (C) Sagittal image of an mRFP-labeled protein trap zebrafish shows a general neural haze with enhancement in specific locations at $100 \times$ magnification (also see Supplemental Movie S5 [showing the spatial location of RFP patterning], available online at www.liebertonline.com). 


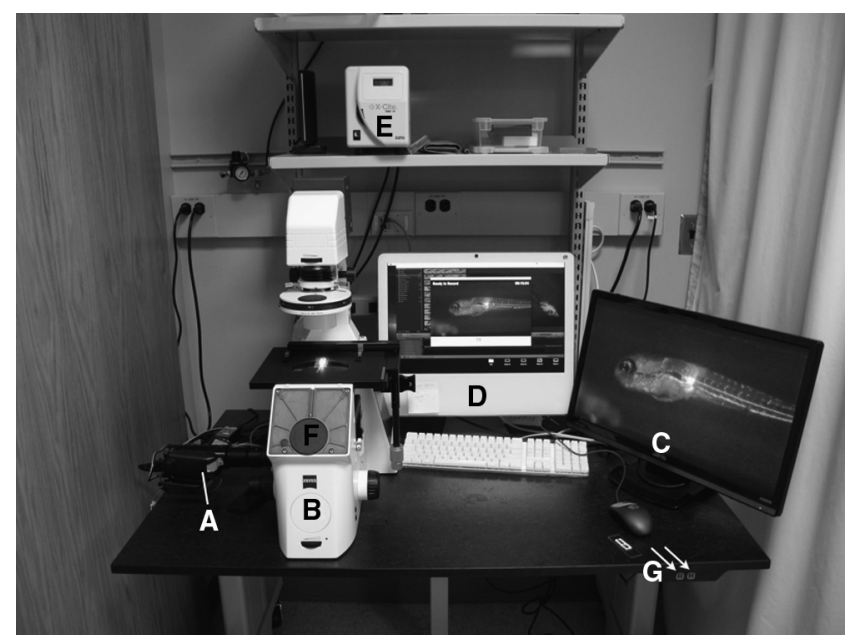

FIG. 5. Ease-of-use microscope-the "headless" microscope setup. (A) A Sony HDR-HC9 high-definition video camera is attached to the camera port of the (B) Zeiss inverted microscope to facilitate a greater ability to view, record, and present visual data. (C) A high-definition monitor allows for reduced eyestrain and ease of presentation to other workers. (D) Attaching the video camera to a computer allows for direct recording of the sample in a purely digital format. (E) $\mathrm{X}$-Cite light source allows for fluorescent microscopy. (F) Removal of the eye-pieces of the Zeiss inverted microscope allows for a reduction in the light bleaching of the sample and provides a less cluttered working area with no obstructions to the stage. $(\mathbf{G})$ The microscope has been placed on a BostonTec adjustable lab table (controls indicated with arrows) to facilitate the ease of movement of the entire scope system for greater ability of access.

\section{Results}

The idea to put a specimen into a capillary tube is not a unique one $\mathrm{e}^{2,12-15}$ with references to similar approaches dating to the late 1920s (see Ref. ${ }^{14}$ and citations therein). Such a method addresses the holding aspect of photography, facilitating the alignment for potentially a full $360^{\circ}$ view of the specimen. However, without modification this approach added major distortion to the light path, especially near the periphery, making the image unfit for data analysis or publication (Fig. 2A; arrows). Using the principles of optics as a guide, the main cause of distortion was determined to be the difference in refractive index between the curved outer surface of the capillary and the air surrounding it, creating a bend in light as described previously. ${ }^{16}$ Normalizing the refractive index of the capillary housing to the liquid surrounding the housing and the fish greatly reduced this effect (cartoon diagrams Fig. 2C and D; example images are Fig. 2E [also see Supplemental Movie S1, available online at www .liebertonline.com] 2F, and 2G). To accomplish a nearly complete refractive index correction, we tested a variety of polymer-based tubes with a refractive index similar to that of water $\left(n=1.33^{17}\right.$ for matching; see Table 1$)$. We evaluated these materials based on four criteria: (1) distortion present, (2) autofluorescence in common wavelengths of light such as deployed in fluorescence microscopy, (3) biocompatibility, and (4) ease of use.

To determine the best imaging housing for the photography of larval zebrafish, we tested FEP tubing $\left(n=1.338^{18}\right)$, perfluoroalkoxy (PFA; $n=1.34^{19}$ ) tubing, and polytetrafuoroethylene (PTFE; $n=1.35^{18}$ ) tubing. Although these tubes perform equally well under most levels of magnification in bright-field applications (FEP shown in Fig. 2E [also see Supplemental Movie S1, available online at www.liebertonline .com] 2F, and 2G; PFA and PTFE not shown), they tend to differ when using red and green fluorescence. We noted that the FEP tubing produced little autofluorescence in either the green fluorescent protein (eGFP filter set; Fig. $2 \mathrm{H}$ [also see Supplemental Movie S2, available online at www.liebertonline.com] and 2I) or red fluorescent protein (such as an mRFP filter set; Fig. 2J [also see Supplemental Movie S3, available online at www.liebertonline.com] and $2 \mathrm{~K}$ ) channels. In contrast, PFA and PTFE tubing showed a higher level of autofluorescence especially with dimmer transgenic fish lines. In addition to the polymer tubing, we also tested a common zebrafish lab reagent, a borosilicate glass capillary $\left(n=1.48^{16}\right)$. For live embryos, the glass capillary was optically matched to a surrounding solution of $50 \%$ sucrose (Table 2 ). This combination provided a clear and crisp image in bright-field applications, but also produced a dark shadow on the edge of the capillary. In fluorescent applications there was no autofluorescence, however. Overall, when photographing a live embryo, we recommend the use of FEP tubing due to its modest cost, lack of autofluorescence, and crisp optical imaging.

SCORE imaging is also an excellent method for imaging fixed embryos, such as commonly generated for WISH. Seventy-five percent glycerol is commonly used to image WISH embryos and is a suitable optical imaging solution using borosilicate glass capillaries (Cartoon, Fig. 3A). Use of the SCORE method on fixed samples is ideal for obtaining images in both the sagittal (Fig. 3B [also see Supplemental Movie S4, available online at www.liebertonline.com], 3C, and 3D) and coronal (Fig. 3E) planes. Additionally, the intricacies of the expression pattern can be displayed in alternative planes of view. For example, if one were to be studying the nephric duct staining shown by pax $2 a$ using WISH, the sagittal view does not show both ducts, and the coronal view is obstructed by additional neural staining (Fig. $3 \mathrm{~B}-\mathrm{E}) . \mathrm{A} \sim 30^{\circ}$ rotation counterclockwise view off the coronal plane (Fig. 3F), however, provides the optimal view of the nephric ducts (Fig. 3F, arrows). Use of the SCORE method also allows for a full rotation of a single embryo to be captured using a video camera attached to the microscope (Fig. 3B [also see Supplemental Movie S4, available online at www.liebertonline.com]). If needed, a precise angle of rotation can be attained with the use of a mechanical aid to ensure reproducibility.

The SCORE method is also a simple and efficient system for creating high-quality wide-field images using standard microscopes at $50 \times$ and $100 \times$ magnifications. With the ease and speed of preparation of samples, this procedure, for example, allows the user to quickly obtain maximum intensity projections of $z$-stacks in the major planes (sagittal, Fig. 4A; dorsal, Fig. 4B) using a single zebrafish and an ApoTome Grid Microscope. The image was of high quality up to $100 \times$ magnification using this imaging setup (sagittal, Fig. $4 \mathrm{C}$ [also see Supplemental Movie S5, available online at www .liebertonline.com]). The greatest limitation to deploying the SCORE method of imaging for whole-animal imaging at higher magnification is the available working distance of most common objectives. 


\section{Discussion}

The key to the SCORE approach is optical correction of an easy specimen housing method. This approach could be applied to chemical genetic testing and other related high-throughput screens. In addition, the use of this method with smaller capillaries could be used for imaging single cells, especially over time with a biocompatible housing solution. Deploying larger capillaries opens the door to the imaging of many other specimens, including synthetic samples where a full $360^{\circ}$ viewing would be desirable. Finally, the housing method developed here could be adapted for use in precisely aligning fixed samples for downstream study such as histological preparation.

Applying the SCORE method at higher magnification is largely limited by two issues-the distortion by air between the samples' cover slip and the lens, and the moderate to low working distance of traditional lenses, including oil immersion optics. One potential option would be the use of optical imaging solution compatible immersion lenses, such as those that work with glycerol and those that work with water. The main limitation will be the thickness of the specimen in relation to the working distance of the objective.

Finally, the ready ability to generate high resolution and multimedia content for scientific articles challenges the contemporary publishing paradigm. The current patch solution is normally a form of supplementary material provided by the publisher, the authors, or both. Instead, we strongly feel that moving forward means the use of a new generation of scientific format that directly integrates such imaging data in the main manuscript.

\section{Acknowledgments}

The authors thank the staff of the Mayo Zebrafish Core Facility for excellent animal care and maintenance. The authors also thank Joel Ash, Dan Heieren, Chi-Bin Chein, and Keith Cheng and anonymous reviewers for their valued input throughout the process of this article. Funding for this work was provided by the Mayo Foundation and by NIH Grants GM63904 and DA14546 to S.C.E.

\section{Author Contributions}

A.M.P., K.J.C., and S.C.E. had the initial idea of the imaging procedure. AMP wrote the initial protocol. V.M.B. and N.J.B. tested and modified the protocol for use with fixed embryos and structured illumination microscopy, respectively. D.B. developed the R14.5 vector, which was injected by J.J.E., to generate the tested transgenic. A.M.P. wrote the article and was aided by all contributing authors.

\section{Disclosure Statement}

No competing financial interests exist.

\section{References}

1. Bryson-Richardson RJ, Berger S, Schilling TF, Hall TE, Cole NJ, Gibson AJ, et al. FishNet: an online database of zebrafish anatomy. BMC Biol 2007;5:34. (Online.)

2. Buckley CE, Goldsmith P, Franklin RJ. Zebrafish myelination: a transparent model for remyelination? Dis Models Mech 2008;1:221-228.
3. Kimmel CB, Ballard WW, Kimmel SR, Ullmann B, Schilling TF. Stages of embryonic development of the zebrafish. Dev Dyn 1995;203:253-310.

4. Sabaliauskas NA, Foutz CA, Mest JR, Budgeon LR, Sidor AT, Gershenson JA, et al. High-throughput zebrafish histology. Methods 2006;39:246-254.

5. Westerfield M. The Zebrafish Book. A Guide for the Laboratory Use of Zebrafish (Danio rerio), 4th edition. Eugene, OR: University of Oregon Press, 2000.

6. Davidson AE, Balciunas D, Mohn D, Shaffer J, Hermanson S, Sivasubbu $\mathrm{S}$, et al. Efficient gene delivery and gene expression in zebrafish using the Sleeping Beauty transposon. Dev Biol 2003;263:191-202.

7. Lawson ND, Weinstein BM. In vivo imaging of embryonic vascular development using transgenic zebrafish. Dev Biol 2002;248:307-318.

8. Traver D, Paw BH, Poss KD, Penberthy WT, Lin S, Zon LI. Transplantation and in vivo imaging of multilineage engraftment in zebrafish bloodless mutants. Nat Immunol 2003;4:1238-1246.

9. Pickart MA, Klee EW, Nielsen AL, Sivasubbu S, Mendenhall EM, Bill BR, et al. Genome-wide reverse genetics framework to identify novel functions of the vertebrate secretome. PLoS One 2006;1:e104.

10. Sivasubbu S, Balciunas D, Davidson AE, Pickart MA, Hermanson SB, Wangensteen KJ, et al. Gene-breaking transposon mutagenesis reveals an essential role for histone H2afza in zebrafish larval development. Mech Dev 2006;123:513529.

11. Petzold AM, Balciunas D, Sivasubbu S, Clark KJ, Bedell VM, Westcot SE, et al. Nicotine response genetics in the zebrafish. Proc Natl Acad Sci U S A 2009;106:18662-18667.

12. Preibisch S, Ejsmont R, Rohlfing T, Tomancak P. Towards digital representation of Drosophila embryogenesis. Proc fifth IEEE Int Symp Biomed Imaging 2008:324-327.

13. Funfak A, Brosing A, Brand M, Kohler JM. Micro fluid segment technique for screening and development studies on Danio rerio embryos. Lab Chip 2007;7:1132-1138.

14. Moroz P. Microscopy and manipulation of cells in elastic capillaries. Trans Am Microsc Soc 1994;113:412-414.

15. Zackson SL. Cell clones and segmentation in leech development. Cell 1982;31:761-770.

16. Budwig R. Refractive index matching methods for liquid flow investigations. Exp Fluids 1994;17:350-355.

17. Schiebener P, Straub J, Levelt Sengers JMH, Gallagher JS. Refractive index of water and steam as function of wave length, temperature and density. J Phys Chem Ref Data 1990;19:677-717.

18. Seferis JC. Refractive Indicies of Polymers. Polymer Handbook, 4th edition, VI/574, VI/5, John Wiley and Sons, 1999.

19. Kerbow DL, Seperati CA. Physical Constants of Fluoropolymers. Polymer Handbook, 4th edition, V/43, John Wiley and Sons, 1999.

Address correspondence to: Stephen C. Ekker, Ph.D. Department of Biochemistry and Molecular Biology Mayo Clinic 201 1st St., SW Guggenheim 13-42C Rochester, MN 55905

E-mail: ekker.stephen@mayo.edu 